\title{
Treatment of heart failure in the elderly: Which drugs are essential and which should be avoided
}

\author{
Giampaolo Scorcu, Annarita Pilleri \\ Cardiovascular Department, Cardiac Evaluation Unit, G. Brotzu Hospital, Cagliari, Italy
}

\begin{abstract}
With improved health care and with population aging, heart failure (HF) has become a common disease among the elderly and is one of the principal causes of mortality in elderly age. But the pharmacological management of HF in the elderly has still not yet been defined, as the clinical context is complicated by comorbidities, and differs from that of younger adults. In general, elderly patients with HF should be treated according to current guideline recommendations, for which ACE-I, beta-blockers and anti-aldosterones constitute the cornerstone of therapy. Interesting prospects are opening up with the use of new drugs such as neprilysin inhibitors, which appear to reproduce in the elderly the positive effects observed in the young adult population, and ivabradine, which may substitute the traditional use (now probably obsolete) of digitalis. Currently, however, treatment of HF in elderly patients is characterized by insufficient drug titration and by a habitual underuse of the recommended therapies - this is partly due to prescription inertia and in part to the negative effect of polypharmacotherapy on patient adherence. Even if HF therapy is similar in older and younger patients, the presence in older patients of more comorbidities, and frailty, functional status, and socio-environmental factors related to aging require a multidisciplinary approach to care and, above all, an additional assessment aimed at personalizing the treatment.
\end{abstract}

\section{Introduction}

Heart failure (HF) is one of the principal causes of morbidity and mortality in elderly patients [1]. Despite the high prevalence and ever rising incidence, elderly patients are underrepresented as a category in large randomized clinical trials, and there are to date no specific guide-

Corresponding author: Giampaolo Scorcu, Struttura Semplice Dipartimentale Consulenza e Valutazione Cardiologica, Ospedale G. Brotzu, Piazzale Alessandro Ricchi 1, 09134 Cagliari, Italy. E-mail: giampaolo.scorcu@tin.it

Key words: Heart failure; elderly patient; pharmacological therapy.

Received for publication: 24 April 2018

Accepted for publication: 11 May 2018

(C) Copyright G. Scorcuz and A. Pilleri, 2018

Tipografia PI-ME Editrice, Italy

Monaldi Archives for Chest Disease 2018; 88:948

doi: 10.4081/monaldi.2018.948

This article is distributed under the terms of the Creative Commons Attribution Noncommercial License (by-nc 4.0) which permits any noncommercial use, distribution, and reproduction in any medium, provided the original author(s) and source are credited. lines for this age-group, the only references to the elderly being limited to the sections devoted to "gaps in evidence". This limitation is all the more serious because HF management differs in the elderly from that in younger patients in many respects, both as regards general characteristics and in specific regard to therapy. Even if in the very elderly, as is often noted, quality of life is more important than simply prolonging it, the main objectives of HF therapy are to alleviate symptoms, maintain or improve functional capacity and quality of life, preserve independence and lengthen survival. This statement is conditioned by the fact that in elderly patients $\mathrm{HF}$ is associated with a high burden of comorbidity, the cause-and-effect relation of which is still not completely clear. Moreover, there is a huge difference between the "average" geriatric patient and the average patient in a large clinical trial: it is not so easy to utilize evidence-based medicine (EBM) in treating elderly patients with $\mathrm{HF}$ because this population is characterized by marked heterogeneity in terms of lifestyle, personal goals and prospects, and by a close link between HF and the cognitive-psychological profile, whose deterioration is an important independent risk factor for adverse events. Considering just the pharmacological aspect, the response to medications can be different in an elderly patient with respect to a younger one; drug interactions and side effects can be higher; comorbidities often necessitate the administration of polypharmacy, as a consequence of which treatment adherence is reduced making the treatment less effective.

Even if there is rarely a "cure" for HF in the elderly population and notwithstanding the fact that many of the problems that geriatricians encounter in their clinical practice have not been - and are unlikely to be - investigated in controlled trials, there is no plausible reason to exclude the elderly from the use of HF drugs, and so current guideline recommendations [2] need to be generalized to include also this patient subgroup.

\section{Treatment of heart failure with reduced systolic} function

\section{Angiotensin-converting enzyme inhibitors and AT-1 angiotensin II receptor blockers}

Angiotensin-converting enzyme inhibitors (ACE-I) have demonstrated their ability to reduce mortality and morbidity in patients with decreased left ventricular (LV) systolic function [3,4], and so unless contraindications or intolerance are present they should be used in all symptomatic patients. Although no recent data are available on the use of ACE-I or AT-1 angiotensin II receptor blockers (ARBs) in elderly patients with reduced systolic function, their use is essential in HF management regardless of age. It is widely demonstrated that in clinical practice the majority of patients receive suboptimal doses of ACE-I [5]; on the contrary, the dose should be titrated up to the maximum tolerated dose in order to obtain an adequate inhibition of the renin-angiotensin-aldosterone system 
(RAAS), paying particular attention to the secondary effects of the drug and to the coexistence of eventual comorbidities. ARBs represent the alternative to ACE-I in the case of their contraindication or intolerance [2]. In special circumstances, a combination of ACE-I and ARBs can be considered, but the association is not recommended in elderly patients due to a high risk of hypotension, deterioration of renal function and hyperkalemia.

\section{Beta-blockers}

Beta-blockers reduce mortality and morbidity in patients with reduced symptomatic systolic function also during treatment with ACE-I and diuretics [6-8]. They should be initiated in clinically stable patients, gradually increasing the dose up to the maximum tolerated dose; in patients with acute HF, the use is advisable only after clinical stabilization. They are particularly recommended in patients with a history of myocardial infarction and asymptomatic systolic dysfunction, to reduce the risk of hospitalization for acute $\mathrm{HF}$ and risk of mortality. The beta-blockers which have the best evidence for proven efficacy and tolerability are bisoprolol, carvedilol and metoprolol [9]. Carvedilol is generally well tolerated in the elderly, including in the over- 80 agegroup, although to a lesser extent in those with advanced New York Heart Association (NYHA) class, low diastolic pressure, obstructive pulmonary disease or concomitant use of amiodarone. In studies comparing the beta-blocking effect at 24 hours, no significant difference was found between metoprolol and atenolol concerning their use in the elderly, although metoprolol has shown a better tolerability than atenolol. Likewise, no significant difference was found between carvedilol and bisoprolol in elderly patients with systolic dysfunction. Bisoprolol has been associated with a better beta-blocking effect and better tolerability in patients with chronic respiratory disease [10]. In contrast, carvedilol, due to its additional blocking of the alpha receptor and greater effect of vasodilation, could be preferable in the absence of significant obstructive pulmonary disease, especially when strict blood pressure control is necessary.

\section{Mineralocorticoid receptor antagonists}

Mineralocorticoid receptor antagonists (MRAs), potassium-sparing diuretics, reduce mortality and incidence of hospitalizations especially in patients with advanced NYHA class, and their benefit has been clearly demonstrated also in elderly patients with HF and reduced systolic function [11]. Despite their proven efficacy, inexplicably, they are underused, their role being seen as a "limiter" of the potassium loss induced by loop diuretics. The MRAs currently in use are spironolactone and eplerenone. It is not clear if these two agents are interchangeable or not, but particular care is needed when they are used in patients with renal failure and in those with serum potassium levels greater than $5 \mathrm{mmol} / \mathrm{L}$.

\section{Loop diuretics}

Diuretics must be titrated in order to reduce signs and symptoms of pulmonary and systemic venous congestion and to maintain euvolemia in patients with $\mathrm{HF}$ with reduced systolic function, but their effects on mortality have never been investigated in randomized clinical trials. It appears from a recent meta-analysis that loop diuretics and thiazides are able to reduce the risk of mortality and deterioration of HF compared to placebo and improve functional capacity [12]. However, due to the frequent side effects such as worsening of renal function and electrolyte disorders, it has to be used, whether singly or in combination, at the lowest possible dose.

\section{Angiotensin II AT-1 receptor/neprilysin inhibitors}

LCZ696 is the progenitor of a new therapeutic class of agents acting on the RAAS which combines the action of an ARB (valsartan) with that of a neprilysin inhibitor (sacubitril). Sacubitril, as a pure inhibitor of neprilysin, promotes the activation of the natriuretic peptide system while increasing the activity of the quinine system, while the blockade of the AT1 receptor by valsartan makes it possible for angiotensin II to interact with the AT-2 receptors, with added positive effects for the decompensated patient. In the PARADIGM-HF [13] study, the effect of sacubitril/valsartan on mortality and morbidity of HF with reduced contractile function was compared to that of ACE-I. Sacubitril/valsartan (97/103 mg bid) was superior to ACE-I (enalapril $10 \mathrm{mg}$ bid), drastically reducing cardiac mortality by $20 \%$ and hospitalizations for acute HF by $21 \%$ at the 3 -year follow-up, with a good overall safety profile. The combination maintained its efficacy also in the over 65 years age-group. In general, sacubitril/valsartan was safe and well tolerated and although hypotension was more frequent, particularly in elderly patients, there was no increase in the rate of suspension even in this subgroup of the population.

\section{$I_{f}$ channel inhibitors: Ivabradine}

Ivabradine is a specific blocker of the $I_{\mathrm{f}}$ channels of the sinoatrial node which causes a reduction in heart rate without inducing any negative inotropic effect. In the SHIFT [14] study, the addition of ivabradine to optimal therapy with beta-blockers, ACE-I and MRA in HF patients with reduced systolic function $(\mathrm{EF}<35 \%)$ and heart rate $\geq 70$ bpm resulted in a significant positive outcome on the primary combined end-point of cardiovascular death and hospitalization for decompensation. Concerns about its use in association with beta-blockers in elderly patients at risk of sinus node dysfunction or symptomatic bradycardia have been dispelled by the demonstration that ivabradine maintains its positive effect without reducing the safety profile. The incidence of severe side effects did not vary substantially from that of younger patients, the only difference being that achieving the target dose in elderly patients was more difficult due to a greater tendency to bradycardia.

\section{Digoxin}

Digoxin can be used to reduce the morbidity correlated to HF with reduced systolic function and in the forms with atrial fibrillation its importance has not changed as a means of modulating heart rate. However, its traditional use as a first step in patients with sinus rhythm and $\mathrm{HF}$, in the beta-blocker era and with the emerging role of ivabradine, is probably now coming to an end. In fact, in patients with sinus rhythm enrolled in the Digitalis Investigation Trial (DIG) study [15], its noneffect on mortality clearly emerged, although there was an associated improvement in patients' quality of life and a significant reduction in hospital admissions. A trend towards increased mortality has been reported - although the finding is still controversial - in patients with plasma digoxin levels between 1 and $2 \mathrm{ng} / \mathrm{ml}$ compared to those with levels of $0.5-1 \mathrm{ng} / \mathrm{ml}$ [16]. For this reason, the use of digitalis in patients with HF in sinus rhythm should be limited to situations in which other drugs are insufficient, with particular attention in elderly patients and in patients with reduced renal function.

\section{Combination of hydralazine and isosorbide dinitrate}

There is no clear evidence supporting the use of this combination therapy in patients with HF with reduced systolic function. The clinical usefulness of this combination is based on the findings of a small trial 
conducted exclusively in men before the spread of ACE-I and betablockers [17]. Historically, the association hydralazine/isosorbide dinitrate has shown an improvement in survival in the black (Afro-American) ethnic population, particularly in the elderly subgroup, but no benefit in the White ethnic group. However, at 6 months after randomization, just over $50 \%$ of patients were still able to tolerate drug administration due to the development of hypotension. The results of these findings are difficult to transfer to patients of other ethnic groups. The routine use of this combination in addition to standard treatment is therefore not recommended due to the risk of hypotension; and the eventual use in addition to beta-blockers, ACE-I or ARBs and MRAs should in any case be reserved only for the black subgroup of the population.

\section{Treatment of heart failure with preserved systolic function}

According to the latest European guidelines, the diagnosis of $\mathrm{HF}$ with preserved systolic function requires an $\mathrm{LVEF} \geq 50 \%$ [2]. Age is a strong predictor of $\mathrm{HF}$ with preserved systolic function - in fact its prevalence is notably higher in elderly than in younger patients [18]. Probably the diagnosis, especially in the elderly population, is underestimated, and a normal contractile function does not necessarily signify a more favorable prognosis with respect to $\mathrm{HF}$ with reduced contractile function. To date, no clinical studies exist demonstrating the efficacy of routine therapy in HF with reduced systolic function [2]. Nevertheless, symptomatic treatment with the use of diuretics and the treatment of cardiovascular comorbidities (hypertension, atrial fibrillation and coronary artery disease) and non-cardiovascular ones (diabetes, renal insufficiency, anemia, COPD, obesity) are a key factor in the management of HF with preserved systolic function $[19,20]$. Even though no treatment has conclusively demonstrated the ability to reduce morbidity or mortality in patients with HF with preserved systolic function, nevertheless, given that these patients are often elderly and highly symptomatic, an important goal of therapy may be to alleviate symptoms and improve quality of life [21]. The potential efficacy of ARNIs also in HF with preserved systolic function represents an interesting prospect for elderly patients. In this regard, randomized clinical trials are already underway in patients with diastolic HF, with death and rehospitalization as the primary outcome, and the results could provide the first conclusive demonstration of the efficacy of a drug in the treatment of this type of HF, typical of the elderly, and with a similar impact on prognosis and quality of life as HF of the systolic type.

\section{Conclusions}

The pharmacological treatment of $\mathrm{HF}$ has made extraordinary progress in the last thirty years thanks to the introduction of ACEI/ARBs, beta-blockers and MRAs, which have drastically reduced HF mortality independently of the disease severity and still today represent the cornerstone of the therapy for this syndrome. It is still not clear, though, whether this can be extended to include the subpopulation of the elderly, whose clinical context is characterized by a dynamic interaction between heart disease, aging, comorbidities, frailty, disability, functional status and socio-environmental factors. Population aging, the exponential increase in the prevalence and incidence of cardiovascular diseases and of HF, associated with the marked heterogeneity of older patients and the presence of multiple comorbidities are in fact generating a pathology with a distinct clinical profile of its own, i.e. a 'cardiogeriatric syndrome'. For this reason, optimal management of $\mathrm{HF}$ in the elderly requires a multidisciplinary approach and its goal is to tailor treatment - both pharmacological and non-pharmacological - to the individual patient. Currently, treatment of HF in elderly patients is characterized by widespread under-utilization of the recommended therapies and suffers from the lack of clinical trials carried out on this particular segment of the population. Subgroups of the elderly population such as the over-80s are in fact totally devoid of specific therapeutic references, and a clinical picture such as $\mathrm{HF}$ with preserved systolic function, which inevitably will be diagnosed more and more in the future, has not yet received adequate attention in the scientific literature. HF is associated with an unfavorable prognosis especially in older people, and there is a need for further research to develop more effective strategies for the prevention and treatment of chronic heart failure and to reduce the individual and social burden of this disease in the decades to come.

\section{References}

1. Rodriguez F, Wang Y, Johnson CE, et al. National patterns of heart failure hospitalizations and mortality by sex and age. J Card Fail 2013;19:542-9.

2. Ponikowski P, Voors AA, Anker SD, et al. 2016 ESC Guidelines for the diagnosis and treatment of acute and chronic heart failure. The Task Force for the diagnosis and treatment of acute and chronic heart failure of the European Society of Cardiology (ESC). Eur J Heart Failure 2016;18:891-975.

3. The CONSENSUS Trial Study Group. Effects of enalapril on mortality in severe congestive heart failure. Results of the Cooperative North Scandinavian Enalapril Survival Study (CONSENSUS). N Engl J Med 1987;316:1429-35.

4. The SOLVD Investigators. Effect of enalapril on mortality and the development of heart failure in asymptomatic patients with reduced left ventricular ejection fractions. N Engl J Med 1992;327:685-91.

5. Maggioni AP, Anker SD, Dahlstrm U, et al. Are hospitalized or ambulatory patients with heart failure treated in accordance with European Society of Cardiology guidelines? Evidence from 12440 patients of the ESC Heart Failure Long-Term Registry. Eur J Heart Fail 2013;15:1173-84.

6. Hjalmarson A, Goldstein S, Fagerberg B, et al. MERIT-HF Study Group. Effects of controlled-release metoprolol on total mortality, hospitalizations, and wellbeing in patients with heart failure: the Metoprolol CR/XL Randomized Intervention Trial in congestive Heart Failure (MERIT-HF). JAMA 2000;283:1295-302.

7. Packer M, Coats AJ, Fowler MB, et al. Effect of carvedilol on the morbidity of patients with severe chronicheart failure: results of the Carvedilol Prospective Randomized Cumulative Survival (COPERNICUS) Study. Circulation 2002;106:2194-9.

8. CIBIS-II Investigators and Committees. The Cardiac Insufficiency Bisoprolol Study II (CIBIS-II): a randomised trial. Lancet 1999;353:9-13.

9. Yancy CW, Jessup M, Bozkurt B, et al. 2013 ACCF/AHA guideline for the management of heart failure: a report of the American College of Cardiology Foundation/American Heart Association Task Force on Practice Guidelines. J Am Coll Cardiol 2013; 62:e147-e239.

10. Dungen HD, Apostolovic S, Inkrot S, et al. Titration to target dose of bisoprolol vs. carvedilol in elderly patients with heart failure: the CIBIS-ELD trial. Eur J Heart Fail 2011;13:670-680.

11. Zannad F, McMurray JJ, Krum H, et al. Eplerenone in patients with systolic heart failure and mild symptoms. N Engl J Med 2011;364:11-21. 
12. Faris RF, Flather M, Poole-Wilson PA, et al. Coats AJ. Diuretics for heart failure. Cochrane Database Syst Rev 2012;2:CD003838.

13. McMurray JJ, Packer M, Desai AS, et al. PARADIGM-HF Investigators and Committees. Angiotensin-neprilysin inhibition versus enalapril in heart failure. N Engl J Med 2014;371:993-1004.

14. Swedberg K, Komajda M, Böhm M, et al. Ivabradine and outcomes in chronic heart failure (SHIFT): a randomised placebo-controlled study. Lancet 2010;376:875-885.

15. Digitalis Investigation Group. The effect of digoxin on mortality and morbidity in patients with heart failure. $\mathrm{N}$ Engl $\mathrm{J}$ Med 1997;336:525-533.

16. Vamos M, Erath JW, Hohnloser SH, et al. Digoxin-associated mortality: a systematic review and meta-analysis of the literature. Eur Heart J 2015;36:1831-1838.

17. Cohn JN, Archibald DG, Ziesche S, et al. Effect of vasodilator therapy on mortality in chronic congestive heart failure. Results of a Veterans Administration Cooperative Study. N Engl J Med 1986;314:1547-1552.

18. Oktay AA, Rich JD, Shah SJ. The emerging epidemic of heart failure with preserved ejection fraction. Curr Heart Fail Rep 2013;10:401-410.

19. Senni M, Paulus WJ, Gavazzi A, et al. New strategies for heart failure with preserved ejection fraction: the importance of targeted therapies for heart failure phenotypes. Eur Heart J 2014;35:2797-2815.

20. Ferrari R, Böhm M, Cleland JGF, et al. Heart failure with preserved ejection fraction: uncertainties and dilemmas. Eur $\mathrm{J}$ Heart Fail 2015;17:665-671.

21. Lewis EF, Lamas GA, 0'Meara E, et al. Characterization of healthrelated quality of life in heart failure patients with preserved versus lowejection fraction in CHARM. Eur $\mathbf{J}$ Heart Fail 2007;9:83-91. 